\section{Common bile duct stones: if you can't sweep them, vacuum them!}

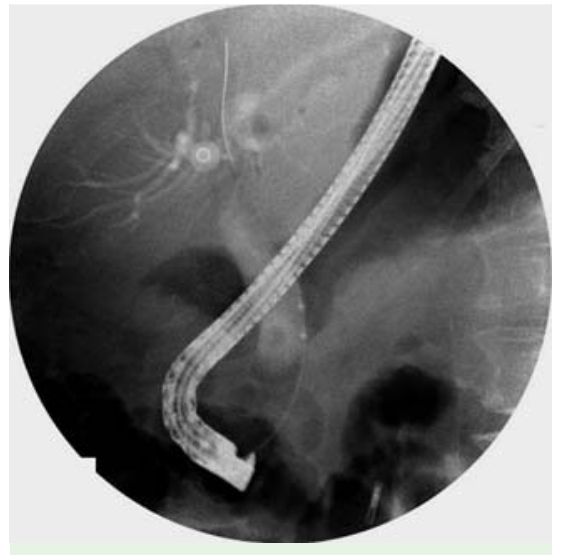

Fig. 1 Cholangiogram taken during endoscopic retrograde cholangiopancreatography (ERCP) showing a markedly dilated common bile duct with a filling defect that could not be

Stone extraction from markedly dilated extrahepatic bile ducts can be challenging because of difficulty in achieving effective balloon apposition with the ductal wall. Studies examining stone extraction strategies in such cases are lacking. We describe this scenario in three patients and technical difficulty. right upper quadrant pain who was found to have cholelithiasis and common bile duct (CBD) dilatation of $20 \mathrm{~mm}$ on imagremoved by repeated balloon sweeps. report a novel technique to overcome this

Patient \#1 was a 64-year-old woman with ing. Laboratory tests revealed elevated results for liver chemistry. Patient \#2 was an 87-year-old woman with Escherichia coli bacteremia and ascending cholangitis who was found on imaging to have a CBD that was dilated up to $23 \mathrm{~mm}$, with an $11-\mathrm{mm}$ filling defect in the distal segment. Patient \#3 was an 11-year-old boy with sickle cell disease who had previously undergone cholecystectomy but exhibited persistent common hepatic duct (CHD) dilatation up to $24 \mathrm{~mm}$ on magnetic resonance cholangiopancreatography (MRCP) in the setting of abnormal liver chemistry results, despite having undergone two prior endoscopic retrograde cholangiopancreatography (ERCP) procedures with sphincterotomy and stent placement.

In the ERCPs of all three patients, filling defects were visualized in large, dilated extrahepatic biliary ducts ( $\bullet$ Fig. 1). Despite multiple attempts, balloon sweeps were not able achieve sufficient apposition with the biliary wall to clear all the retained biliary stones or sludge.

The balloon was therefore advanced to the bifurcation of the left and right intrahepatic ducts. During subsequent balloon sweeps, negative pressure was gently applied to the biliary system using a $60-\mathrm{mL}$ syringe on a wire port to collapse the proximal bile duct ( $\bullet$ Fig.2). This allowed apposition of the balloon and the wall of extrahepatic bile duct, which allowed stones and debris to be successfully swept out by the balloon. Complete clearance of the bile duct was confirmed by an occlusion cholangiogram ( $\bullet$ Fig. 3 ) in patients \#1 and \#3, and by cholangioscopy in patient \#2.

The treatment of these three patients illustrates a novel and successful technique using negative pressure during ERCP to clear difficult-to-remove biliary stones from markedly dilated extrahepatic bile ducts, and one which can be readily employed in practice.

Endoscopy_UCTN_Code_TTT_1AR_2AH

Competing interests: None

\section{Amol Sharma, Abraham Mathew}

Penn State Milton S. Hershey Medical Center, Hershey, Pennsylvania, USA

Bibliography

Dol http://dx.doi.org/

10.1055/s-0034-1391498

Endoscopy 2015; 47: E175

(c) Georg Thieme Verlag KG

Stuttgart · New York

ISSN 0013-726X

\section{Corresponding author}

\section{Amol Sharma, MD}

500 University Drive

Medicine Suite 2400

Hershey

Pennsylvania 17033

USA

asharma6@hmc.psu.edu

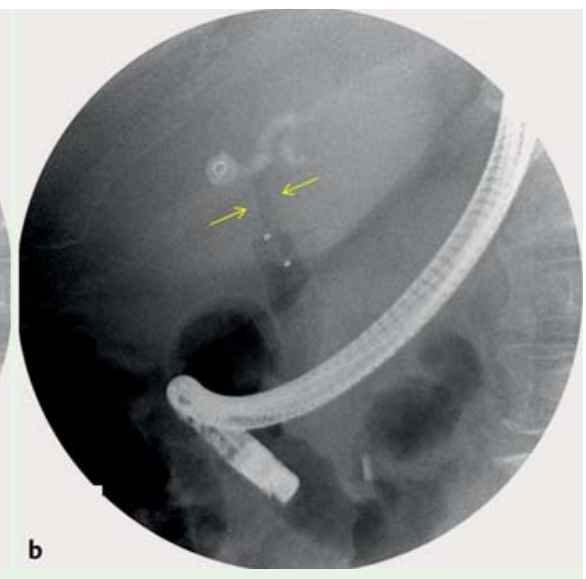

a
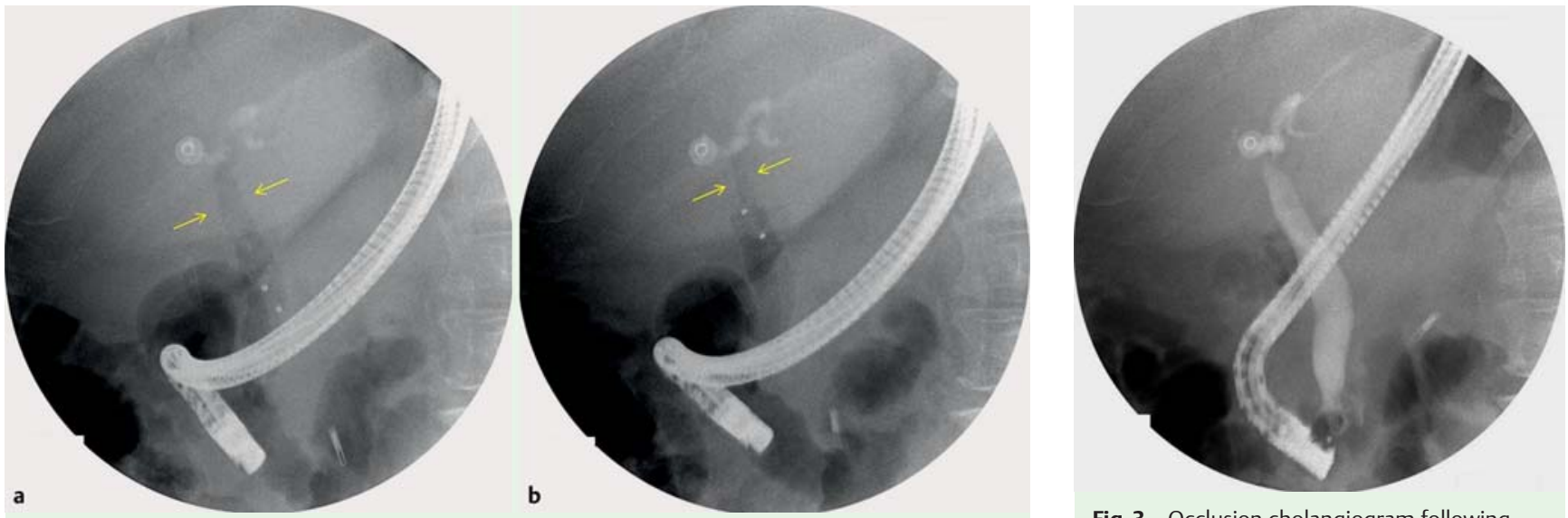

Fig. 3 Occlusion cholangiogram following negative-pressure balloon sweeps showing the common bile duct without any filling defects. 